\title{
Tissue drives lesion: computational evidence of interspecies variability in cardiac radiofrequency ablation
}

\author{
Argyrios Petras $^{1, *}$, Massimiliano Leoni ${ }^{1,2}$, Jose M. Guerra ${ }^{3}$, Johan Jansson ${ }^{1,2}$, and \\ Luca Gerardo-Giorda ${ }^{1}$ \\ ${ }^{1}$ BCAM - Basque Center for Applied Mathematics, Bilbao, Spain \\ ${ }^{2}$ Department of Computational Science and Technology, KTH Royal Institute of \\ Technology, Stockholm, Sweden \\ ${ }^{3}$ Department of Cardiology, Hospital de la Santa Creu i Sant Pau, CIBERCV, \\ Barcelona, Spain \\ *Alameda de Mazarredo 14, 48009 Bilbao, Bizkaia, Spain, apetras@bcamath.org
}

April 16th, 2019

\begin{abstract}
Radiofrequency catheter ablation (RFCA) is widely used for the treatment of various types of cardiac arrhythmias. Typically, the efficacy and the safety of the ablation protocols used in the clinics are derived from tests carried out on animal specimens, including swines. However, these experimental findings cannot be immediately translated to clinical practice on human patients, due to the difference in the physical properties of the types of tissue. Computational models can assist in the quantification of this variability and can provide insights in the results of the RFCA for different species. In this work, we consider a standard ablation protocol of $10 \mathrm{~g}$ force, $30 \mathrm{~W}$ power for $30 \mathrm{~s}$. We simulate its application on a porcine cardiac tissue, a human ventricle and a human atrium. Using a recently developed computational model that accounts for the mechanical properties of the tissue, we explore the onset and the growth of the lesion along time by tracking its depth and width, and we compare the lesion size and dimensions at the end of the ablation.
\end{abstract}

Keywords: radiofrequency catheter ablation, mathematical model, tissue properties, interspecies variability.

\section{Introduction}

Radiofrequency ablation (RFA) is a common treatment for cardiac arrhythmias. Through a catheter advanced into the patient's heart, radiofrequency current is delivered to the tissue which produces resistive heating in the neighborhood of the electrode, while conduction propagates the 
heat to the immediate surrounding tissue. At a temperature of $50{ }^{\circ} \mathrm{C}$ the tissue is irreversibly damaged and a permanent lesion develops [3]. RFA is generally safe, however life-threatening complications can occur, including the formation of thrombi due to blood overheating at $80^{\circ} \mathrm{C}$ and steam pops at temperatures of $100{ }^{\circ} \mathrm{C}$ within the tissue, which are among the most severe complications [3].

Typically, ablation protocols are designed and tested using in-vitro, ex-vivo and in-vivo experiments on animals. Porcine cardiac tissue is commonly used in the experiments to assess the efficacy and the safety of the RFA treatment $[1,5]$. However, the biophysical, mechanical and physiological properties of the porcine cardiac tissue differ from the corresponding human ones [6], thus a direct translation of the experimental results to clinical practice can lead to insufficient treatment or potential life-threatening complications. Computational models can be a valuable asset in the assessment of RFA protocols. The efficacy and safety of the procedure can be directly assessed on simulated human tissue using the reported physical properties in the literature, thus avoiding the translation of data from experiments that use porcine tissue.

In this study, we investigate the interspecies variability in the RFA treatment using a recently developed computational model [5] which includes the mechanical properties of the tissue. We simulate a commonly used ablation protocol on cardiac tissue of two species: porcine and human. Two different ablation sites are considered in the case of the simulated human tissue: an atrium and a ventricle.

\section{Computational model}

\section{$2.1 \quad$ Geometry}

Based on an experimental setup similar to [1], an $80 \mathrm{~mm} \times 80 \mathrm{~mm} \times 80 \mathrm{~mm}$ box is constructed that includes blood (top $40 \mathrm{~mm}$ ), cardiac tissue of thickness $H \mathrm{~mm}$ and an external factors board (bottom $40-H \mathrm{~mm}$ ). Within the blood compartment, the catheter is placed perpendicularly to the tissue at the middle of the box. We consider a 6 -hole electrode with a hemispherical tip and a thermistor inspired by RFA catheters commonly used in clinics, as described in [5]. Figure 1 shows a sample computational geometry for a tissue thickness of $H=20 \mathrm{~mm}$.

Due to the contact with the catheter, the tissue undergoes a mechanical deformation. The vertical deformation is described in [5] and is formulated as

$$
\omega(r)= \begin{cases}\omega_{\max }-\left(R-\sqrt{R^{2}-r^{2}}\right), & r \leq a, \\ \frac{a^{2}}{\pi} \int_{0}^{1} \frac{2 \omega_{\max }-a x \log \left(\frac{R+a x}{R-a x}\right)}{r^{2}-a^{2} x^{2}} d x, & r>a,\end{cases}
$$

where $R$ is the radius of the electrode, $\omega_{\max }$ is the maximum indentation depth, $a$ is the contact radius of the electrode with the tissue and $r$ is the planar distance from the center of the electrode. The indentation depth and contact radius can be computed using the contact force $F$, the Young's modulus $E$ and the Poisson's ratio $\nu$ of the tissue, as follows:

$$
\omega_{\max }=\frac{a}{2} \log \left(\frac{R+a}{R-a}\right),
$$



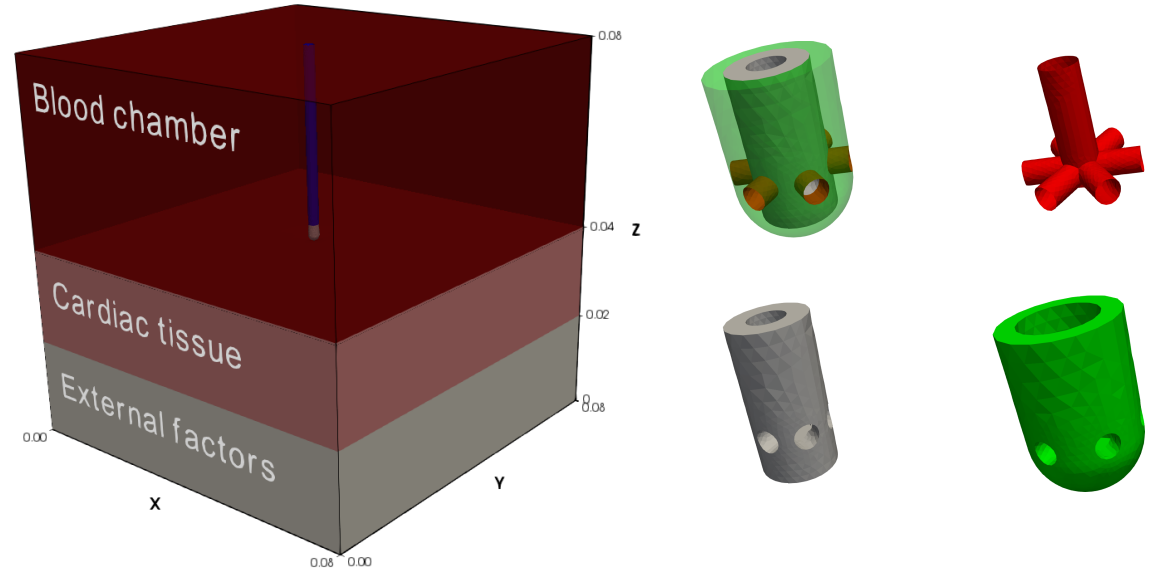

Figure 1: Left: The full computational geometry. Right: The computational tip of the catheter (top left), the saline tubes (top right), the thermistor (bottom left) and the electrode (bottom right).

$$
F=\frac{E}{2\left(1-\nu^{2}\right)}\left(\left(a^{2}+R^{2}\right) \log \left(\frac{R+a}{R-a}\right)-2 a R\right) .
$$

The deformation of the tissue is directly embedded in the construction of the computational geometry.

\subsection{Governing equations}

The blood flow and its interaction with the irrigated saline in the blood compartment $\Omega_{\text {blood }}$ of the geometry are modelled using the incompressible Navier-Stokes equations

$$
\begin{aligned}
\frac{\partial \mathbf{u}}{\partial t}+\mathbf{u} \cdot \nabla \mathbf{u}-\operatorname{div} \sigma(\mathbf{u}, p) & =\mathbf{0} \\
\operatorname{div} \mathbf{u} & =0
\end{aligned}
$$

where $\mathbf{u}$ is the velocity, $\sigma(\cdot, \cdot)$ is the stress tensor and $p$ is the scaled pressure. A constant inflow is considered in one of the sides of $\Omega_{\text {blood }}$ with its corresponding outflow conditions at the opposite side. A constant inflow is also considered from the saline irrigation holes on the electrode flowing radially within $\Omega_{\text {blood }}$. The remaining interfaces of $\Omega_{\text {blood }}$ are equipped with no slip conditions.

A modified Penne's bioheat equation tracks the changes in the temperature of the geometry $\Omega$ over time

$$
\rho c(T)\left(\frac{\partial T}{\partial t}+\mathbf{u} \cdot \nabla T\right)-\operatorname{div}(k(T) \nabla T)=\sigma(T)|\nabla \Phi|^{2},
$$

where $T$ is the temperature, $t$ is the time, $\rho$ is the density, $c(T)$ is the specific heat, $\mathbf{u}$ is the velocity, $k(T)$ is the thermal conductivity, $\sigma(T)$ is the electrical conductivity and $\Phi$ is the electrical potential. A temperature of $22^{\circ} \mathrm{C}$ is assumed on the interface between the irrigation holes and 
the blood compartment $\Omega_{\text {blood }}$ to model the cooling effect of the saline, while insulation boundary conditions are applied on the irrigation tubes and the catheter body. A constant body temperature of $37^{\circ} \mathrm{C}$ is imposed on all outer boundaries of the computational domain $\Omega$.

The spatial distribution of the potential follows a quasi-static equation, augmented with a power constraint for constant power ablations

$$
\left\{\begin{array}{l}
\operatorname{div}(\sigma(T) \nabla \Phi)=0, \\
\int_{\Omega} \sigma(T)|\nabla \Phi|^{2} d x=P,
\end{array}\right.
$$

where $P$ is the power dissipated within the computational domain $\Omega$. A potential $V_{0}$ is applied on the interface of the catheter and the electrode, tuned to match the power $P$ dissipated in the computational domain $\Omega$. The dispersive electrode is placed at the bottom of $\Omega$, where zero potential is imposed. All the remaining surfaces are considered electrically insulated. More details on the computational model can be found in [5].

\subsection{Parameters}

The parameters considered in the computational model are summarized in Table 1. The specific heat $c$, thermal and electrical conductivities $k$ and $\sigma$ are considered temperature dependent within the tissue:

$$
\begin{aligned}
c(T) & =c_{0}\left(1+c_{1}(T-37)\right) \\
k(T) & =k_{0}\left(1+k_{1}(T-37)\right) \\
\sigma(T) & =\sigma_{0}\left(1+\sigma_{1}(T-37)\right)
\end{aligned}
$$

The electrical conductivity of the external effects board $\sigma_{b}$ is tuned to match the initial resistance of the system, and the power delivered to the tissue, which is calculated as follows:

$$
P_{\text {tissue }}=\frac{A_{\text {tissue }} \sigma_{0}^{(\text {tissue })}}{A_{\text {blood }} \sigma_{0}^{(\text {blood })}+A_{\text {tissue }} \sigma_{0}^{(\text {tissue })}} P_{a b l}=: \alpha P_{a b l},
$$

where $A_{\text {tissue }}$ and $A_{\text {blood }}$ are the surface areas of the electrode in contact with the tissue and the blood respectively, $\sigma_{0}^{(\text {tissue) }}$ and $\sigma_{0}^{(\text {blood })}$ are the electrical conductivities of the tissue and the blood at body temperature and $P_{a b l}$ is the total power set by the ablation protocol. The initial resistance of the system is set as $120 \Omega$. More details on the calculation of $\sigma_{b}$ can be found in [5].

In our extensive literature review, no work has been found that addresses differences in biophysical properties on different regions of the human heart. Thus, we assume that the human atrium and ventricle have the same biophysical properties, which are drawn from [2] at body temperature. The temperature dependence of the specific heat and thermal conductivity can be calculated from [10]. No data were found for the dependence of the electrical conductivity of the myocardium on the temperature, thus values for the human liver are considered, since a similar behavior is reported for other species [6]. The mechanical properties for human cardiac tissue are summarized in $[7,9]$.

The thickness of the porcine cardiac wall is considered as $H=20 \mathrm{~mm}$ [4]. Two different substrates are modelled for the simulated human cardiac tissue: an atrium of $H=6 \mathrm{~mm}$ thickness and a ventricle of $H=12.5 \mathrm{~mm}[8,11]$. 
Table 1: The summary of the biophysical and mechanical parameters that appear in the computational model.

\begin{tabular}{l|c|c|c|c|c|c|} 
Parameters & Blood & Electrode & Thermistor & Board & \multicolumn{2}{|c|}{ Tissue } \\
& & & & & Porcine & Human \\
\hline$\rho\left(\mathrm{kg} \mathrm{m}^{-3}\right)$ & 1050 & 21500 & 32 & 1076 & 1076 & 1081 \\
$c_{0}\left(\mathrm{~J} \mathrm{~kg}^{-1} \mathrm{~K}^{-1}\right)$ & 3617 & 132 & 835 & 3017 & 3017 & 3686 \\
$c_{1}\left({ }^{\circ} \mathrm{C}^{-1}\right)$ & - & - & - & - & -0.0042 & -0.0011 \\
$k_{0}\left(\mathrm{~W} \mathrm{~m}^{-1} \mathrm{~K}^{-1}\right)$ & 0.52 & 71 & 0.038 & 0.518 & 0.518 & 0.56 \\
$k_{1}\left({ }^{\circ} \mathrm{C}^{-1}\right)$ & - & - & - & - & -0.0005 & 0.0022 \\
$\sigma_{0}\left(\mathrm{~S} \mathrm{~m}^{-1}\right)$ & 0.748 & $4.6 \times 10^{6}$ & $10^{-5}$ & $\sigma_{b}$ & 0.54 & 0.381 \\
$\sigma_{1}\left({ }^{\circ} \mathrm{C}^{-1}\right)$ & - & - & - & - & 0.015 & 0.015 \\
$\nu(-)$ & - & - & - & - & 0.499 & 0.499 \\
$E(\mathrm{kPa})$ & - & - & - & - & 75 & 40
\end{tabular}

Table 2: The summary of the temperature and lesion size dimensions after the completion of $30 \mathrm{~s}$ of ablation for the simulated tissue considered.

\begin{tabular}{l|c|c|c|} 
& Porcine & Human Ventricle & Human Atrium \\
\hline$D(\mathrm{~mm})$ & 3.76 & 3.35 & 3.75 \\
$W(\mathrm{~mm})$ & 6.73 & 6.56 & 7.42 \\
$D W(\mathrm{~mm})$ & 1.38 & 0.86 & 0.94 \\
\hline$S A\left(\mathrm{~mm}^{2}\right)$ & 0.11 & 0.0 & 0.0 \\
$V\left(\mathrm{~mm}^{3}\right)$ & 103.4 & 92.3 & 124.9 \\
\hline$T_{\max }$ tissue $\left({ }^{\circ} \mathrm{C}\right)$ & 77.7 & 71.9 & 77.3 \\
$T_{\max }$ blood $\left({ }^{\circ} \mathrm{C}\right)$ & 51.5 & 56.1 & 62.0
\end{tabular}

\section{Results}

We simulate a standard constant power ablation protocol of $30 \mathrm{~W}$, with a contact force of $10 \mathrm{~g}$ for a total of $30 \mathrm{~s}$ of ablation. The blood flow protocol is set to $0.1 \mathrm{~m} \mathrm{~s}^{-1}$ and the saline irrigation rate to $17 \mathrm{ml} \mathrm{min}{ }^{-1}$. This ablation protocol is typically used in RFA experiments [1].

We identify the computational lesion by the $50^{\circ} \mathrm{C}$ isotherm contour: the quantities measured are the depth $(D)$, the width $(W)$, the depth at which the maximum width occurs $(D W)$, the surface area of the lesion $(S A)$ and the volume $(V)$. In addition to the lesion size dimensions mentioned above, we track the maximum temperature in the tissue and the blood. Details on the measurement of these quantities can be found in [5].

The computational results are shown in Table 2. The lesion on the human atrium appears to be the biggest in volume, while the smallest is on the human ventricle. The depth in the porcine cardiac tissue and the human atrium are comparable, while the lesion is more shallow in the human ventricle. A bigger difference appears in the measured width. In particular, a difference of nearly $1 \mathrm{~mm}$ is observed between the cases of human atrium and ventricle, while the porcine lesion width 
is comparable with the human ventricle one. The morphology of all three lesions appears to be different, with significant changes in the depth at which the maximum width occurs. Though the maximum width of the lesions on the human specimen appears to be more shallow, there is no surface burning. A small surface burn is present in the case of the porcine cardiac tissue.

The temperature of the tissue is similar in the cases of the porcine cardiac tissue and the human atrium, while it is about $5{ }^{\circ} \mathrm{C}$ lower in the human ventricle. On the contrary, the blood temperature is much lower in the porcine cardiac tissue. The temperature difference with respect to the human tissue is bigger than $10^{\circ} \mathrm{C}$ for the atrium and around $5{ }^{\circ} \mathrm{C}$ for the ventricle.
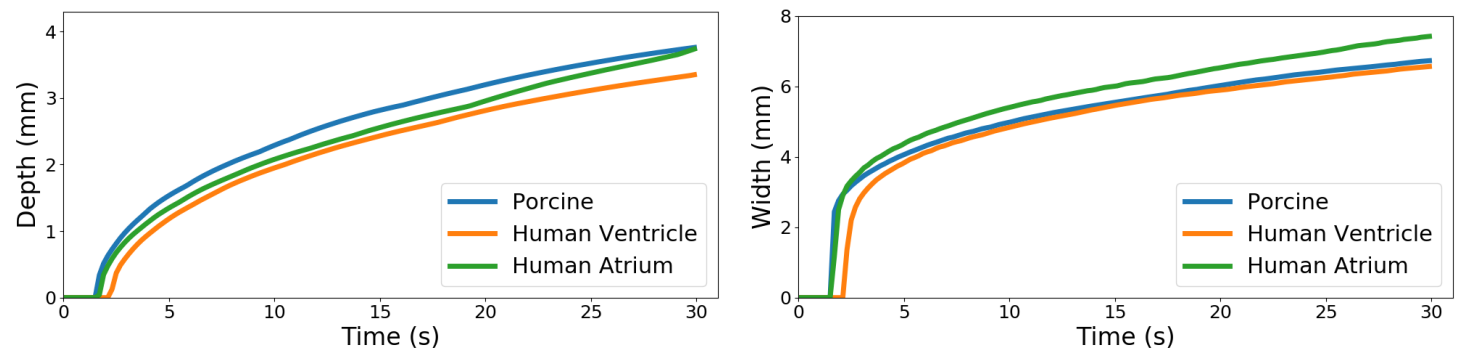

Figure 2: The lesion depth (left) and width (right) over time for the simulated porcine cardiac tissue, human ventricle and human atrium.

To further compare the results on the three types of tissue considered, we explore in Figure 2 the evolution of the width and the depth of the lesion throughout the duration of the ablation. We observe that the lesion is created almost at the same time on the porcine cardiac tissue and the human atrium, while the one in the human ventricle is delayed by $0.8 \mathrm{~s}$. The depth of the lesion in the porcine case is consistently larger than the one in the human tissue. The depth of the lesion in the human atrium case is initially similar to the human ventricle case, but after $20 \mathrm{~s}$ it increases to a depth comparable to the porcine case. On the contrary, the width of the lesion in the human atrium is larger than the other cases throughout the duration of the ablation, while comparable values appear between the human ventricle and the porcine tissue.

Finally, to explore morphological differences of the lesions, we show in Figure 3 the medial and lateral view of the $50{ }^{\circ} \mathrm{C}$ isotherm contour within the tissue at the final time of the ablation. All three lesions appear tilted towards the direction of the blood in the lateral view, while they are symmetric in the medial view, as previously observed in [5]. The lesions in the human tissue appear elongated and less spherical than the ones in the porcine tissue, which is reflected in the difference in the depth of the maximum width in Table 2.

\section{Conclusions}

We explored interspecies variability during RFA treatment, comparing the resulting lesion size dimensions of the ablation process on a porcine cardiac tissue, a human ventricle and a human atrium. Our computational results indicate that the lesion characteristics are different in all three cases. The human atrium provides the largest lesion overall. The depth of the lesion in the porcine cardiac tissue case is larger than the one on the human tissue throughout the ablation process. 

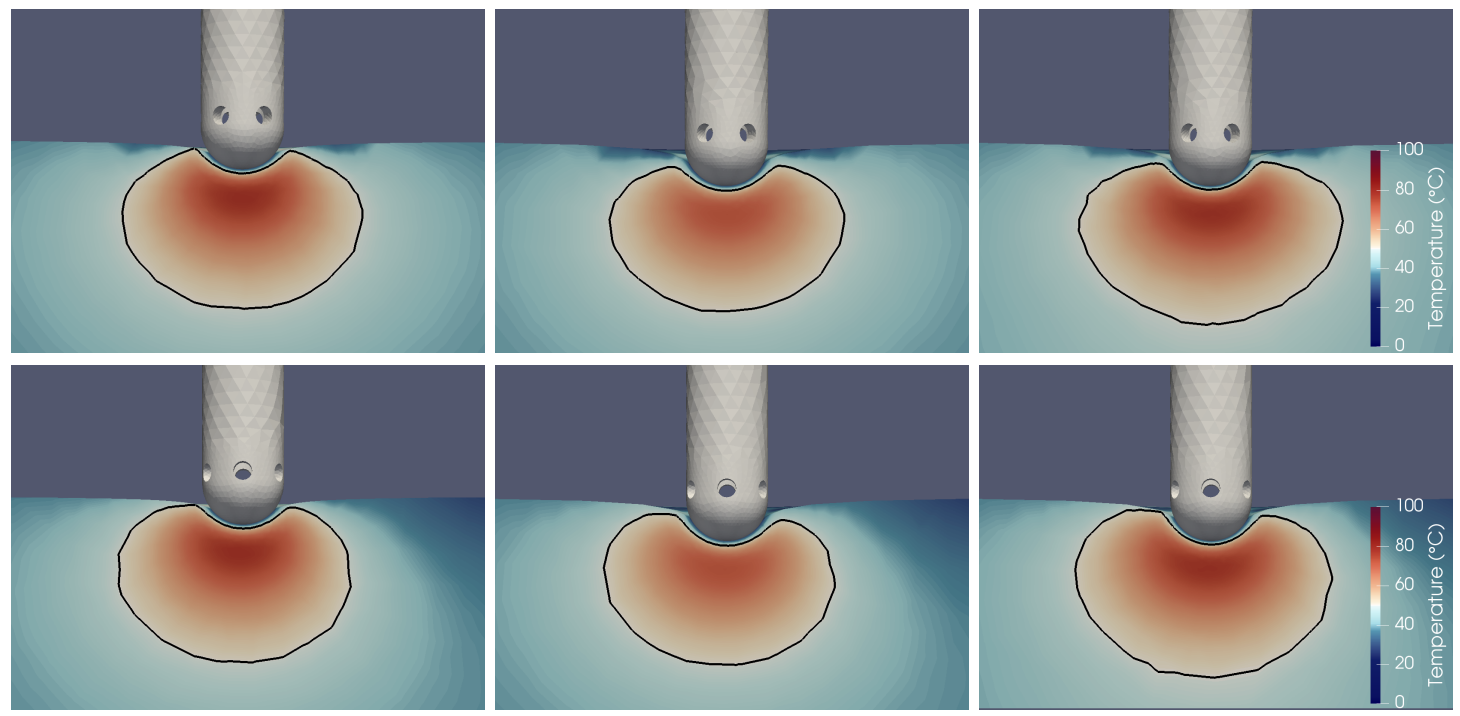

Figure 3: The medial (top) and lateral (bottom) view of the lesion for porcine cardiac tissue (left), human ventricle (middle) and human atrium (right) at the final time of the ablation.

Thus, a direct application to a human patient of a protocol based on the achievement of a specific lesion depth in porcine tissue would result in too small lesions and insufficient treatment in cases that transmural lesions are targeted.

The width of the lesion in the human atrium is at least $0.7 \mathrm{~mm}$ larger than the other two cases. In addition, the lesions in the simulated human tissue appear less spherical than the porcine one. A direct translation from porcine data to the human atrium would affect the efficiency of the RFA process, indicating that a larger number of lesions are required when targeting isolation lines in the atrium.

The maximum tissue temperature in the atrium and the porcine tissue are comparable, however there is a difference of more than $10^{\circ} \mathrm{C}$ the maximum blood temperature. Regarding the human ventricle, the tissue temperature is lower than the porcine one, however the maximum blood temperature is about $5{ }^{\circ} \mathrm{C}$ higher. This indicates that the results on porcine tissue can lead to blood overheating and thrombus formation if directly translated to human tissue. On the contrary, steam pops would be avoided as the maximum tissue temperature is overestimated, though the efficiency of the process can be reduced by inducing smaller lesions in the human ventricle.

\section{Acknowledgements}

This work is supported by the Basque Government through BERC 2019-2021 and by the Spanish Ministry of Economy and Competitiveness MINECO through BCAM Severo Ochoa excellence accreditation SEV-2017-0718. 


\section{References}

[1] Jose M Guerra, Esther Jorge, Silvia Raga, Carolina Gálvez-Montón, Concepción AlonsoMartín, Enrique Rodríguez-Font, Juan Cinca, and Xavier Viñolas. Effects of open-irrigated radiofrequency ablation catheter design on lesion formation and complications: In vitro comparison of 6 different devices. Journal of Cardiovascular Electrophysiology, 24(10):1157-1162, 2013.

[2] P.A. Hasgall, E. Neufeld, M.C. Gosselin, A. Klingenböck, and N. Kuster. Itis database for thermal and electromagnetic parameters of biological tissues. Version 3.0, 2015.

[3] Shoei K Stephen Huang and Mark A Wood. Catheter Ablation of Cardiac Arrhythmias E-book. Elsevier Health Sciences, 2014.

[4] Si-kwang Liu, Y.T. Chiu, J.J. Shyu, Stephen M. Factor, Redman Chu, J.H. Lin, H.L. Hsuo, P.R. Fox, and P.C. Yang. Hypertrophic cardiomyopathy in pigs: quantitative pathologic features in 55 cases. Cardiovascular Pathology, 3(4):261-268, 1994.

[5] Argyrios Petras, Massimiliano Leoni, Jose M Guerra, Johan Jansson, and Luca GerardoGiorda. A computational model of open-irrigated radiofrequency catheter ablation accounting for mechanical properties of the cardiac tissue. arXiv preprint arXiv:1810.09157, 2018.

[6] Christian Rossmann and Dieter Haemmerich. Review of temperature dependence of thermal properties, dielectric properties, and perfusion of biological tissues at hyperthermic and ablation temperatures. Critical Reviews in Biomedical Engineering, 42(6), 2014.

[7] Jens Rump, Dieter Klatt, Jürgen Braun, Carsten Warmuth, and Ingolf Sack. Fractional encoding of harmonic motions in MR elastography. Magnetic Resonance in Medicine, 57(2):388-395, 2007.

[8] A-L. Sjögren. Left Ventricular Wall Thickness Determined by Ultrasound in 100 Subjects without Heart Disease. Chest, 60(4):341-346, 1971.

[9] Mihai Strachinaru, Johan G. Bosch, Bas M. van Dalen, Lennart van Gils, Antonius F.W. van der Steen, Nico de Jong, Marcel L. Geleijnse, and Hendrik J. Vos. Cardiac Shear Wave Elastography Using a Clinical Ultrasound System. Ultrasound in Medicine ${ }^{3}$ Biology, 43(8):1596-1606, 2017.

[10] J. W. Valvano, J. R. Cochran, and K. R. Diller. Thermal conductivity and diffusivity of biomaterials measured with self-heated thermistors. International Journal of Thermophysics, 6(3):301-311, 1985.

[11] John Whitaker, Ronak Rajani, Henry Chubb, Mark Gabrawi, Marta Varela, Matthew Wright, Steven Niederer, and Mark D O'Neill. The role of myocardial wall thickness in atrial arrhythmogenesis. Ep Europace, 18(12):1758-1772, 2016. 